\title{
What Causes Different Sentiment Classification on Social Network Services? Evidence from Weibo with Genetically Modified Food in China
}

\author{
Youzhu Li ${ }^{1}$, Xianghui Gao ${ }^{1}$, Mingying Du ${ }^{1,2}$, Rui He ${ }^{1}$, Shanshan Yang ${ }^{3, *}$ and Jason Xiong ${ }^{4}(\mathbb{D}$ \\ 1 College of Public Administration, Huazhong Agricultural University, Wuhan 430070, China; \\ liyz@mail.hzau.edu.cn (Y.L.); gaoxh@webmail.hzau.edu.cn (X.G.); mingyingdu136188@163.com (M.D.); \\ hr19930301@webmail.hzau.edu.cn (R.H.) \\ 2 College of Accounting, Wuchang Institute of Technology, Wuhan 430065, China \\ 3 College of Media and Art Design, Wuhan Donghu University, Wuhan 430212, China \\ 4 Walker College of Business, Appalachian State University, Boone, NC 28608, USA; xiongjj@appstate.edu \\ * Correspondence: shanshanyang2009@163.com; Tel.: +86-15971505658
}

Received: 9 January 2020; Accepted: 7 February 2020; Published: 12 February 2020

\begin{abstract}
Background Genetic Modification (GM) refers to the transfer of genes with known functional traits into the target organism, and ultimately the acquisition of individuals with specific genetic traits. GM technology in China has developed rapidly. However, the process is controversial; thus, future development may be hindered. China has become the world's largest importer of GM products. Research on the attitudes towards GM food in China will help the government achieve sustainable development by better understanding and applications of the technology. (2) Methods This research utilizes data from Sina Weibo (microblog), one of the biggest social network services (SNS) in China. By using the self-created Python crawler program, comments related to the genetically modified food in the People's Daily account are analyzed. Sentiment classifications are analyzed via multivariate logistic regression. (3) Results Based on the factor analysis, theme type characteristics, the propagation characteristics, the body information characteristics, and the comment characteristics have different degrees of influence on the user's emotional distribution. (4) Conclusion Practical implications and conclusions are provided based on the results at the end.
\end{abstract}

Keywords: information systems; genetic modification; sentiment classification; logistic regression; China; sustainable development

\section{Introduction}

Genetic Engineering, Genetic Modification (GM) or Genetic Manipulation is the transfer of genes with known functional traits such as high yield, stress resistance, disease resistance, and improved nutritional quality into the target organism through modern scientific and technological means so that the recipient organism can add new functions based on the original genetic characteristics [1]. Compared to the traditional hybrid breeding technology, GM can obtain the excellent traits that the former is difficult to produce, so it has great prospects. Since its birth, it has been viewed by most countries as the highlight of science and technology and the development of modern agricultural technology [2,3]. However, at the same time as the rapid development of GM technology, there are controversies surrounding various aspects of GM technology. The public opinion environment directly affects the research and development of GM technology in China [4,5]. At the same time, with the increasing participation of the public in the network, various new media platforms, including Sina Weibo [6], are widely used and become the carrier for public opinions and expressions [7]. There are many stakeholders involved in GM technology. This also involves a wide range of topics, and 
the influence of public opinion is largely impacted by the "group polarization" [8,9]. Therefore, whenever a new GM-related topic emerges in the network, a large amount of commentary information will be generated on the social network service platform, and these comments will reflect the user's sentient classification toward GM related technologies. Sentiment classification refers to the emotional classifications of user's attitudes, opinions, and positions on a certain product or a certain thing [10-12]. It is usually expressed in subjective text information. The sentiment analysis is applied to analyze and identify text information to determine whether the expression is positive, neutral, or negative.

Exploring the sentiment classification of users in comments and further analyzing their influencing factors can help decision-makers better understand, guide, and optimize online public opinion. This research utilizes Sina Weibo as the source for data mining, analyzes the emotional tendency of relevant comments, and builds a model to analyze the influence of various factors. Based on the results, policymakers can guide and improve the GM related regulations, improve the public's acceptance of GM technology, and promote the sustainable development of GM technology in China.

\section{Literature Review}

\subsection{GM Technology and Public Perception}

GM technology has been hot for a long time. The academia has done a lot of research around it, mainly focusing on the public's perception, acceptance and influencing factors on GM technologies or food, mostly based on off-line investigations. Scott [13] studied the attitudes of life scientists and laypeople to genetically modified foods separately, and found that the attitudes of life scientists are more positive than laypeople, while laymen tend to regard genetically modified foods as dangerous foods with little benefit. Flavio [14] surveyed more than 900 Italian households and found that consumers have very little preference for genetically modified food. Kaya and Konar [15] surveyed Turkish households and show that although it is believed that GM technologies and foods are economically beneficial, consumers still present a negative attitude in view of factors such as health, environment, and biodiversity. Ghasemi, et al. [16] surveyed Iranian agricultural professionals and found that their knowledge about GM foods was at a low level. They also found low recognition had a negative impact on their consumption behavior. For China, Huang and Peng [4] conducted a follow-up survey of Chinese consumers related to GM. They found that during the period 2002-2012, the proportion of consumers who thought that GM foods were unsafe was increased by more than $30 \%$, and about half of consumers did not have an opinion on this issue. Han, et al. [17] found that consumer acceptance of GM foods is generally low, but Chinese consumers from developed regions are more willing to accept and pay for GM foods. However, cotton farmers are positive about GM cotton due to significant economic benefits.

In terms of influencing factors, McComas, et al. [18] studied the factors that American consumers support for genetically modified foods, and the results include consumer perceptions of human or environmental risks, perceived benefits, and objective knowledge about GM. Öz, et al. [19] surveyed young consumers in the millennial generation of the United States and found that the higher the level of education, especially the higher the level of biotechnology knowledge, the easier it is to accept the genetic modification. In addition, the smaller the food risk, the easier it is to accept, and men support GM technology more than women. Bukenya and Wright [20] investigated the factors affecting the acceptability of GM tomatoes, the results indicate that factors affecting consumer decision-making include attitude toward the use of genetic modification technology in food production, opinion about labeling, and consumer perceptions about the safety of GM foods. Curtis et al. [4] surveyed Romanian consumers found that income levels and risk perception are the main factors affecting purchasing decisions. For Chinese consumers, De Steur, Gellynck, Feng, Rutsaert and Verbeke [5] studied the factors affecting the willingness of Chinese consumers to pay for genetically modified rice, and concluded that the objective knowledge, information acquisition and perceived benefits of genetically modified organisms will affect their willingness to pay. Zhu and Xie [21] focused on the impact of 
respondents' risk and benefits knowledge on their attitudes towards GM foods. Huang and Peng [4] found that personal and family characteristics, such as gender, education: food allergy experience and resident city size, will affect consumers' perceptions of GM food safety. In addition, the public's perception of genetic modification is also influenced by other subjects. Han, Zhou, Liu, Cheng, Zhang and Shelton [17] believed that increasing the acceptance of genetic modification in China requires the educational efforts of government officials, the media and scientists. In particular, government agencies and public media have more effective educational efforts on the scientific facts and safety of genetically modified foods. Huang and Peng [4] found in the investigation that negative media coverage affects GM technology. Lu, et al. [22] suggested that social trust can effectively reduce public awareness of the risks of genetically modified foods.

\subsection{Emotional Analysis}

In recent years, the application value of subjective texts has been explored by more and more scholars. With emotional analysis as a hot topics, there have been many studies in this area. Some scholars have studied the calculation of emotional tendency and improved the accuracy of the analysis. Park and Kim [23] extended the word set by collecting synonyms and antonyms in three different online English dictionaries and established a classification dictionary for emotional classification. $\mathrm{Ai}$, et al. [24] proposed an emotion classification algorithm combining dictionary and integrated classifier. Liu, et al. [25] proposed a method of emotional tendency analysis which based on the view of the context, improved the accuracy. Asghar, et al. [26] proposed a general framework that combines cognitive-based emotion theory with computational techniques based on sentiment analysis to detect and classify emotions in natural language texts and can be used in any domain. There are also some researches on microblog text. De-Yang, et al. [27] improved the microblog emotional analysis method based on key sentences and proposed a topic-related keyword extraction algorithm. Huang, et al. [28] proposed a new emotion taxonomy method, including basic emotions and complex emotions in microblog text, and constructed an emotional corpus to achieve. $\mathrm{Xu}$, et al. [29] through feature extraction and feature selection, classified Weibo posts into fine-grained emotion classes and used hierarchical classification to improve the performance of the classifier.

Many studies further apply emotional analysis to different domains. Wang, et al. [30] studied the emotional tendency in Massive Open Online Courses (MOOCs). They tracked the learner's emotion through the semantic analysis model. It was used to analyze students' acceptance of the courses and the graduation probability at different stages. Zhu, et al. [31] studied the role of specific emotions in conceptual classification and found that emotive participants had a greater tendency than neutral mood participants to group concepts. Sun, et al. [32] explored the emotional tendencies in the field of e-business and detected customer emotions by analyzing adverbs in emotional expressions. The results can be used to improve related processes in e-business. Hu, et al. [33] found that the number of online reviews and the feedback emotions of user comments can affect product sales by studying the relationship between book sales and the number of comments and user comments. Topal and Ozsoyoglu [34] conducted an emotional analysis of movie scores and comments on the IMDb website to generate emotional maps of the movies, making it easy for users to make choices.

In addition, some scholars have studied the emotions on social platforms. Habibi, et al. [35] based on Twitter, explored the relationship between student learning styles and emotional tendencies. It turned out that different emotional tendencies correspond to different learning styles. Nofer, et al. [36] predicted stock returns by extracting user sentiment levels from Twitter. Tumasjan, et al. [37] predicted sentiment trends in political events (government, elections, security and defense, and health insurance) by processing and classifying the data in Twitter. Zhao, et al. [38] proposed a deep event-emotion analysis system on Microblogs, explored the causes of different emotions in heated events. Kramer, et al. [39] experimented with Facebook users, found that face-to-face interaction and non-verbal cues are not necessary for emotional contagion, emotional contagion also exists in the network. Stieglitz et al. [32] studied the relationship between emotion and information dissemination 
in social media and found that emotional Twitter messages were forwarded more frequently and faster compared to neutral ones. Goel and Thareja [40], based on Twitter data, analyzed user sentiment and found that fear is the least expressed and sadness is the most expressed emotion. Fan, et al. [41] explored the emotional associations of users in Weibo. They found that the emotions of anger are more relevant than the emotions of joy; that is, angry emotions are more likely to spread.

It can be seen that scholars have done a lot of research related to genetic modification. The exploitation of emotional orientation and social platform text has become a hot topic in academic circles. These studies have deepened our understanding of genetic modification and influencing factors, and have played an important role in further mining microblog information for emotional orientation analysis. However, in the case of transgenic related research, researchers use the traditional questionnaire method, and we are now in the era of big data. The network has a large number of reports and commentary information related to genetic modification, which is a huge resource for related research. In the aspect of emotional orientation research, for the topic of high public sensitivity and obvious emotional polarization, no scholars have used relevant data to analyze emotional sentiment. This study combines genetic modification, emotional orientation, and big data to compensate for the lack of research. Firstly, the Python crawler program is used to obtain the data, and then the artificial intelligence platform is used to judge the sentiment orientation. Finally, using the characteristic variables unique to Weibo, the model is constructed to analyze the factors affecting the sentiment tendency comprehensively and objectively.

\section{Research Methodology}

\subsection{Data Source and Collection}

As an emerging social platform, Weibo attracts more and more users by its unique values in timeliness and interactivity. It has become an important platform for people to receive information and comment, and it is also the main platform for emotional research. By the third quarter of 2019, Sina Weibo's monthly active users reached 497 million. People's Daily account in Sina Weibo has more than 100 million followers. This demonstrates the influence and authority of this Weibo account. This study utilizes the Python-based crawler program to collect the content containing the "Genetically Modified" field published by People's Daily since 2013 and the comments and commentator information under each Weibo and obtained a total of 20,837 messages.

The data collection process followed the steps below. Step 1. We entered the collection page according to the Uniform Resource Locator (URL) address. The URL address was based on Google's search from Sina Weibo People's Daily account and contains the URL of the "Genetically Modified" field messages. Step 2. We crawled the relevant texts one by one for the content of the page, including the date of publication of Weibo, the content of the text, the number of forwards, the number of comments, and the number of likes. Step 3. We crawled the comments under each Weibo, and collected the user page by commenting on the user link to crawl the relevant information of the user. Step 4 . We cycled through the second and third steps until all the data had been crawled. The Python statement is shown in Figure 1 below.

\subsection{Data Cleaning}

The raw data contained some noise data that were not related to the research. In order to reduce its impact on the research results, the data needed to be cleaned so that the research results were more reliable. This work was done manually by the following steps. (1) We removed the microblogs that were not closely related to the topic. Some microblogs only had the word "genetically modified" in the text, but the content was not about genetically modified events. For example, "media fusion is a genetic engineering project". This is not relevant to the research. (2) We removed data with missing values. In the process of collecting data, since some users change their ID name or have logged off the microblog, only the user comment content and the original ID name could be obtained, 
and the user data were missing. (3) We removed irrelevant contents from the comments, including advertisements, links starting with "http", simply @other users or just forwarding a Weibo without commenting. These comments did not reflect the user's emotions. After data cleaning, 12,031 valid data were finally obtained.

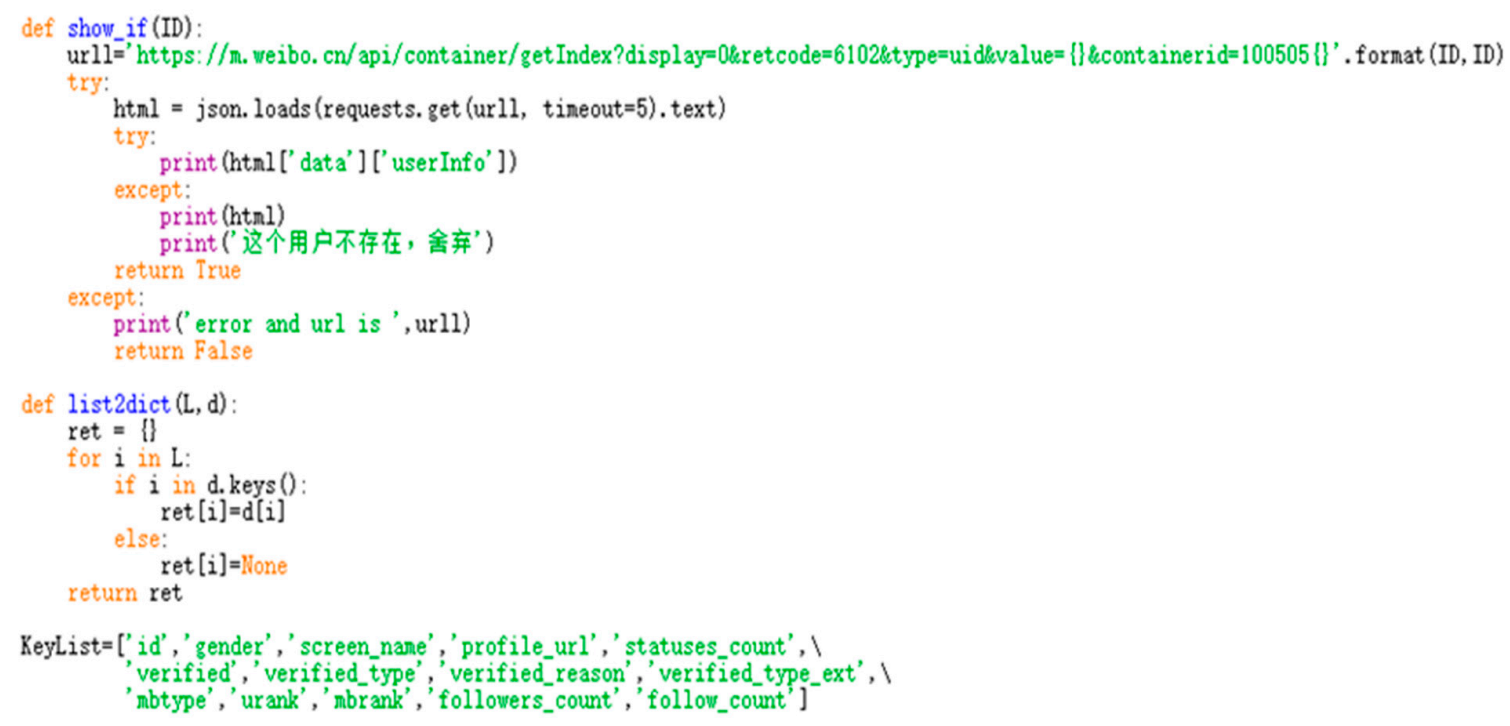

Figure 1. Python based crawler program.

\subsection{Data Processing and Variables Selection}

\subsubsection{Determinations of Emotional Classifications}

In recent years, artificial intelligence (AI) has been applied in many fields. This paper uses the AI open platform developed by Baidu to determine the sentiment orientation. The natural language processing (NLP) module can provide a rich class library of Chinese word segmentation sentiment orientation analysis, and based on the deep learning method, it establishes sentence-level, entity-level, and chapter-level multi-granularity complete analysis tasks. The sentence-level granularity is based on the Bi-LSTM classification method. The system better captures the information expressed by the emotional polarity in the context, and the effect is greatly improved compared with the traditional method. Through the API (application programming interface), the corpus containing a large amount of text can be used to judge the emotional polarity category (positive, negative, neutral) of the text containing subjective information, and the corresponding confidence is given.

The purpose of utilizing the API is to provide an application so that developers can call the function of this program without accessing the source code. The API used in this study is a sentiment orientation analysis interface developed by Baidu AI platform. It can realize the emotional tendency analysis of the comment data only by calling it at the computer terminal. The returned parameters include positive probability, negative probability, and emotional polarity result. When outputting results, 0,1 , and 2 represent negative, neutral, and positive emotions, respectively. Table 1 below shows an example of the output result.

\subsubsection{Independent Variable Selection and Coding}

A Weibo, or microblog, mainly consists of two parts: The main text and comments. Therefore, when the variables are selected, the variables are determined according to the existing research and our data crawling methods, including the main topics, the propagation effect variables, the main body and the comment variables. 
Table 1. Example of Output Result.

\begin{tabular}{cccc}
\hline Comments & $\begin{array}{c}\text { Positive } \\
\text { Emotions } \\
\text { Probability }\end{array}$ & $\begin{array}{c}\text { Negative } \\
\text { Emotions } \\
\text { Probability }\end{array}$ & $\begin{array}{c}\text { Emotional } \\
\text { Polarity } \\
\text { Category }\end{array}$ \\
\hline $\begin{array}{c}\text { Unscrupulous special interest group } \\
\text { I expect more discussion in genetically modified area } \\
\text { Ignorance can't cover science, and genetic } \\
\text { modification will benefit mankind. }\end{array}$ & 0.0787637 & 0.921236 & 0 \\
& 0.467929 & 0.532071 & 1 \\
\hline
\end{tabular}

The main theme is the type of topic from the microblog. The characteristics of the microblog propagation effect include forwarding number, number of comments, and number of likes. The characteristics of microblog text include the length of the microblog text, the number of pictures, whether there is forwarding content, Is there a video, a link, and a @symbol? The comment feature variable is mainly related to the reviewer's characteristics, including gender, number of fans, number of followers, number of Weibo posts, ratings, and whether the reviewer is authenticated. In addition, Weibo has the function of praise for comments. Each Weibo is often the top of some popular comments. These are the more praised comments. Hot comments indicate that the opinions expressed by them are more recognized by others and more affect the emotions of other reviewers, so the number of comments per comment is also used as a variable.

Among the above variables, categorical variables are created based on whether there is forwarding content, whether there is a video, whether there is a link, whether there is a @ symbol, and whether the

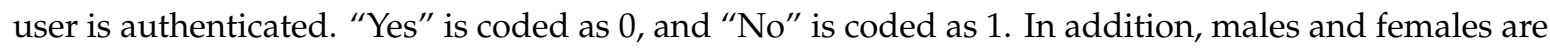
coded as 0 and 1, respectively. In terms of the type of microblogging topic, since the GM topic involves many aspects, the current research only focuses on one aspect, such as "security" and "information disclosure", and there is no exhaustive topic classification. Therefore, this paper classifies the acquired microblogs, uses the NLPIR semantic analysis system to extract keywords, and combines relevant literature and expert opinions to finally determine the types of GM microblog topics including food safety, government regulation, information disclosure, policy system, and public. Six categories of rights, science and rumors, coded from 1 to 6 . Due to the limited nature of the data, other topics such as "environment" and "ethics" are not covered. The topics and corresponding microblog features are shown in Table 2.

Table 2. Topic types and corresponding microblog text features.

\begin{tabular}{|c|c|}
\hline Themes of The Topics & Microblog Text Features \\
\hline Food Safety & Report on Genetically Modified (GM) food safety incidents \\
\hline Government Regulation & $\begin{array}{l}\text { Exposing adverse events in GM regulation, reporting on government } \\
\text { agencies' attitudes toward GM management, measures taken, etc. }\end{array}$ \\
\hline Information Disclosure & $\begin{array}{l}\text { Government discloses information on approved genetically modified } \\
\text { varieties, genetically modified safety certificates, etc. }\end{array}$ \\
\hline Policy & $\begin{array}{l}\text { Reports on systems and regulations for genetically modified planting, } \\
\text { import and export, labeling, etc. }\end{array}$ \\
\hline Public interest & Emphasis on public rights to know and choose genetically modified foods \\
\hline Popular Science & $\begin{array}{l}\text { Scientific knowledge about genetic modification, reciting the scientific } \\
\text { statements of experts and rumoring GM rumors }\end{array}$ \\
\hline
\end{tabular}

\section{Data Analysis and Results}

\subsection{Model Selection}

The dependent variable of this study is the emotional tendency, which is divided into three categories, negative emotion, neutral emotion and positive emotion with no order. Therefore, we 
choose an unordered multiple logistic regression model for analysis. A logistic regression model is suitable for solving nonlinear regression problems, determining the degree of influence of multiple independent variables on the dependent variable, the model is mature, and the accuracy is also high. It is a powerful tool for dealing with categorical variables. In addition, the model does not require that each variable obey a multivariate normal distribution, so it can be used more widely. The multiple logistic regression model analyzes the influence of each factor by comparing the selected category and the reference category in each variable, and the result indicates the probability of the event. The formula is:

$$
\operatorname{Ln}\left(\frac{P_{j}}{P_{i}}\right)=\alpha_{j}+\sum_{k=1}^{n} \beta_{j k} x_{k}
$$

The reference category is represented by $i . P_{j}$ represents the conditional probability of occurrence of the $j$-th event. $\operatorname{Ln}\left(\frac{P_{j}}{P_{i}}\right)$ presents the natural logarithmic of the ratio of selected category $j$ to reference category $i . \beta_{j k}$ is the regression coefficient of the $k$-th independent variable, $x_{k}$ is the independent variable, and $\alpha_{j}$ is a constant term.

\subsection{Model Validation}

In this study, SPSS 24.0 was used for model testing and analysis. In order to optimize the model, the independent variables were forward-introduced, and the independent variables that were not statistically significant were eliminated. Table 3 shows the fitting information of the model, and it is a test for whether the partial regression coefficients of the respective variables in the model are all zero. It can be seen that the $-2 \log$-likelihood is $23,747.556$ only at the intercept, and finally decreases to $15,023.424$ after adding the variable, the significance level is 0.000 , and the significance level is less than 0.05 . This indicates that at least one of the partial regression coefficients of the respective variables is not zero, and the overall model fitting effect is good.

Table 3. Model Fitting Information.

\begin{tabular}{ccccc}
\hline Model & $\mathbf{- 2}$ log-Likelihood & Chi-Square & Degrees of Freedom & Significance Level \\
\hline Intercept only & $23,747.556$ & & & \\
Final & $15,023.424$ & 8724.132 & 40 & 0.000 \\
\hline
\end{tabular}

Table 4 shows the statistically significant independent variable likelihood ratio test results left by the model. The result excludes the "whether there is a link" variable, and the significance levels of the remaining variables are all 0.000 , indicating that these variables all have a significant impact on the effective tendency studied in this paper.

\subsection{Descriptive Statistics and Analysis}

As there are many variables and different types of data in this research, the descriptive statistics analysis will help better understand the data and conduct further analysis.

Table 5 presents the descriptive statistical analysis results. It can be seen that the proportion of negative emotions is the highest among the three types of emotions. $40.6 \%$ of users do not show obvious positive or negative emotions on transgenes, while positive emotions are only one-third of negative emotions, and the environment of transgenic public opinion is not optimistic. Therefore, it is more important to explore the factors that affect the public's emotional tendencies and optimize the public opinion of GM networks. 
Table 4. Model Likelihood Ratio Test.

\begin{tabular}{ccccc}
\hline Variables & -2 log-Likelihood & Chi-Square & $\begin{array}{c}\text { Degrees of } \\
\text { Freedom }\end{array}$ & $\begin{array}{c}\text { Significance } \\
\text { Level }\end{array}$ \\
\hline Intercept & $15,023.424^{\text {a }}$ & 0.000 & 0 & \\
Topics & $15,416.815$ & 393.391 & 10 & 0.000 \\
No. of Forwarding & $15,073.891$ & 50.467 & 2 & 0.000 \\
No. of Comments & $15,041.064$ & 17.640 & 2 & 0.000 \\
No. of Likes & $15,077.240$ & 53.816 & 2 & 0.000 \\
Text Length & $15,073.914$ & 50.490 & 2 & 0.000 \\
No. of Pics & $15,040.299$ & 16.875 & 2 & 0.000 \\
Video Included or Not & $15,071.226$ & 47.802 & 2 & 0.000 \\
@ included or Not & $15,049.828$ & 26.404 & 2 & 0.000 \\
Forward Included or Not & $15,061.247$ & 37.823 & 2 & 0.000 \\
No. of Followers & $15,051.200$ & 27.776 & 2 & 0.000 \\
No. of Following & $15,388.593$ & 365.169 & 2 & 0.000 \\
No. of Weibo & $15,144.611$ & 121.187 & 2 & 0.000 \\
Level & $16,121.430$ & 1098.006 & 2 & 0.000 \\
Gender & $16,971.637$ & 1948.213 & 2 & 0.000 \\
Verified Account or Not & $15,548.067$ & 524.643 & 2 & 0.000 \\
Likes Received & $15,394.726$ & 371.302 & 2 & 0.000 \\
\hline
\end{tabular}

Chi-square statistics are the -2 log-likelihood difference between the final and simplified models. The simplified model is formed by omitting an effect from the final model. The null hypothesis is that all parameters of the effect are zero. ${ }^{a}$ Because omitting this effect does not increase the degree of freedom, this simplified model is equivalent to the final model.

Table 5. Emotional Tendency Results.

\begin{tabular}{ccc}
\hline & Sample Size & Percentage \\
\hline Negative Emotions & 5413 & $45.0 \%$ \\
Neutral Emotions & 4879 & $40.6 \%$ \\
Positive Emotions & 1739 & $14.5 \%$ \\
Total & 21031 & $100 \%$ \\
\hline
\end{tabular}

Table 6 presents the descriptive statistical results of continuous variables in this study. It can be seen that the average value of reposts and comments on "genetically modified" Weibos are higher than the likes, and the maximum value is more than 5000. After further analysis of the microblogs issued by People's Daily, it was found that Weibo has a high variable of transmission effect. It can be seen that the microblog events related to the GM have a greater impact, and will not receive more praise from users. For users, the number of followers and posts shows the influence and activity of a user. As shown in Table 6, the standard deviation (S.D.) is much higher than other variables. This indicates that the influence and activity of each user are quite different. At the same time, it was found that the average number of users' followers and posts was relatively high, which indicated that most of the users who participated in the discussion of GM microblog topics had some influence and activity. They also paid more attention to social topics such as GM.

Table 6. Continuous Variables Descriptive Statistics.

\begin{tabular}{cccccc}
\hline & Sample Size & Minimum & Maximum & Average & S.D. \\
\hline No. of Forwarding & 12,031 & 263 & 5978 & 1932.80 & 1796.650 \\
No. of Comments & 12,031 & 129 & 5543 & 1072.29 & 1222.870 \\
No. of Likes & 12,031 & 6 & 1108 & 292.48 & 289.878 \\
No. of Followers & 12,031 & 0 & $56,172,775$ & $11,155.87$ & $531,252.120$ \\
No. of Following & 12,031 & 0 & 6220 & 423.63 & 520.527 \\
No. of Weibo & 12,031 & 0 & 187,077 & 4318.94 & $10,146.385$ \\
Level & 12,031 & 0 & 48 & 24.24 & 9.161 \\
Likes Received & 12,031 & 0 & 119 & 0.22 & 1.860 \\
\hline
\end{tabular}


In addition, the statistics of the user's gender and authentication status were analyzed. It was found that there were 9013 male users, accounting for $74.9 \%$, and 3018 female users, only one-third of male users. There are only 716 authenticated users, accounting for $6 \%$, and they have rarely participated in GM related discussion on Weibo.

\section{Research Model Analysis and Results}

\subsection{Regression Analysis}

The negative emotion tendency is selected as the reference category. Each categorical variable in the independent variables uses the last coding category as the reference category. The final regression results of the model are shown below.

\subsubsection{Neutral Emotion Regression Results}

Based on results from Table 7 , in contrast to negative emotions, the variables that have a significant effect on neutral emotions include food safety, government regulation, and public interest in themes of the topics; No. of Forwarding, No. of Comments, and No. of Likes in Propagation Effect; text length, number of pictures, video included or not and forwarded or not in the Text information characteristics; number of followers, followings, Weibo posts, level, and gender in the comment characteristics. Among them, the value of $\operatorname{Exp}(\mathrm{B})$ represents the ratio of the probability level of the dependent variable under the influence of the independent variable to the probability level of the dependent variable under the influence of the reference variable when the other conditions are the same. A value greater than 1 indicates that the independent variable has a positive effect on the dependent variable, while a value less than 1 indicates a negative effect.

Table 7. Model parameter estimates (reference group = negative sentiment).

\begin{tabular}{cccc}
\hline Types of Variables & Variables & $\begin{array}{c}\text { Neutral Emotions } \\
\text { Exp (B) }\end{array}$ & $\begin{array}{c}\text { Positive Emotions } \\
\text { Exp (B) }\end{array}$ \\
\hline Themes of The Topics & Food Safety & $0.697^{* * *}$ & $0.192^{* * *}$ \\
& Government Regulation & $0.484^{* * *}$ & $0.063^{* * *}$ \\
& Information Disclosure & 1.155 & $1.199^{* * *}$ \\
& Policy & 0.967 & $0.271^{* * *}$ \\
Propagation Effect & Public Interest & $1.250^{* *}$ & $0.490^{* * *}$ \\
& No. of Forwarding & $0.708^{* * *}$ & $0.575^{* * *}$ \\
& No. of Comments & $1.152^{* * *}$ & $0.899^{* *}$ \\
Text information characteristics & No. of Likes & $1.257^{* * *}$ & $1.810^{* * *}$ \\
& Text Length & $1.3011^{* * *}$ & $0.531^{* * *}$ \\
& No. of Pics & $1.114^{* * *}$ & $1.004^{* * *}$ \\
& Video Included or Not & $1.3633^{* * *}$ & $3.143^{* * *}$ \\
& @ included or Not & 1.044 & $1.804^{* * *}$ \\
& Forward Included or Not & $0.650^{*}$ & $4.096^{* * *}$ \\
Comment characteristics & No. of Followers & $9.013^{*}$ & $8.238^{* *}$ \\
& No. of Following & $1.948^{* * *}$ & $2.634^{* * *}$ \\
& No. of Weibo & $2.013^{* * *}$ & $2.447^{* * *}$ \\
& Level & $2.354^{* * *}$ & $4.653^{* * *}$ \\
& Gender & $10.704^{* * *}$ & $52.087^{* * *}$ \\
& Verified Account or Not & $6.658^{* * *}$ & $59.250^{* * *}$ \\
\hline
\end{tabular}

*** $p<0.01 ;{ }^{* *} p<0.05 ; * p<0.1$.

It can be seen that the variables that have a positive impact on neutral sentiment include public interest theme types (1.250), number of reviews (1.152), number of likes (1.257), text length (1.301), number of pictures (1.114), video included or not (1.363), the number of followers (9.013), the number of followings (1.948), the number of posts (2.013), the level (2.354), male users (10.704), and authenticated 
users (6.658). Variables that negatively affect neutral sentiment include food safety (0.694), government regulation (0.484) topic types, retweets $(0.708)$, retweets $(0.650)$, and praise $(0.102)$ for comments.

\subsubsection{Positive Emotion Regression Results}

As can be seen from Table 7, in contrast to negative emotions, the variables that have a significant effect on positive emotions include food safety, government regulation, policy systems, public interest in Weibo topic-type characteristic variables, number of retweets and likes, length of text in Weibo body information feature variables, whether there is a video, whether there is @, whether there is repost content, number of followings, followers, posts, level, gender, whether certified, and likes received for this comment.

Among them, the variables that have a positive impact on positive emotions include the number of likes (1.810) with video (3.142), with @ symbol (1.804), with reposted content (4.096), number of followers' followers (8.238), followers number (2.634), Weibo number (2.447) and rank (4.653), male users (52.087), and authenticated users (59.250). Variables that negatively affect positive emotions are food safety (0.192), government regulation (0.163), policy systems $(0.271)$, public interest theme types (0.490), retweets (0.575), text length (0.531), and comments and likes received (0.688).

\subsection{Results Analysis}

\subsubsection{Weibo Theme Characteristic Variables}

As a comparison between popular science and Weibo, the two types of topics, food safety and government regulation, have a significant negative impact on neutral and positive emotions. This is because when these two topics are generally involved, most of them are negative GM events exposed by the media, such as "Yonghe Soymilk was detected and returned with a genetically modified ingredient" or "Three of the five bags of rice in Wuhan are genetically modified rice". GM was a sensitive topic, and some negative events appeared, which caused serious adverse effects. At that time, a wave of public opinion was formed and the Internet was full of negative speech. The theme of the policy system has a negative impact on positive emotions. On the one hand, it is caused by the lack of credibility of our government for a long time. After a series of negative events, the public has become increasingly distrustful of the government. On the other hand, the government has a problem in policy formulation. Issues such as non-publicity, opaqueness, and lack of public participation have led to public resistance to some GM policies. The microblog of the public interest theme has a positive impact on neutral emotions, but still has a negative impact on positive emotions. The reason is that when it comes to public rights, most people's daily calls for the right to know and choose about genetic modification, whether to eat or not to eat and the comments are mostly the public's demand for the right to choose and know. Negative emotions are not as strong as the previous three types of Weibo.

\subsubsection{Weibo Propagation Effect Characteristic Variables}

The number of forwarding, comments and likes is the most intuitive manifestation of the spread of a microblog. The larger the value, the greater the influence of the Weibo. Among them, the number of forwarding has a negative impact on both neutral and positive emotions, which indicates that there are more negative sentiments on Weibo, and the number of reposts is also higher. The so-called "good things don't go out, bad things spread a thousand miles" negative events will get more attention and have a greater impact. The number of likes has a positive effect on both neutral and positive emotions, which is consistent with our cognition. Likes indicate that users like Weibo content, so more positive emotions will appear in the comments. Regarding the number of comments, it was found in the research that perhaps due to the special nature of the GM topic, many Weibo comment sections showed as "This Weibo bans comments", so this variable did not truly reflect the spread. 


\subsubsection{Weibo Text Information Characteristic Variables}

The variables in this type are the amount of information that can be displayed in the content of the microblog body, which helps to better convey information and increase influence. Text length has a positive effect on neutral emotions but has a negative effect on positive emotions. The probable cause is that longer content will convey more information, and people will think more when reading, instead of venting without thinking, which will help alleviate some negative emotions, but overall the text length is longer. It is often the narration and reporting of some negative events, which does not increase the public's positive emotions. Including pictures and videos in Weibo will reduce negative emotional tendencies and have positive effects on neutral and positive emotions when there are videos. This result is because in the information crawled, many pictures and videos are more specific descriptions of the body content, which is convenient for users to understand the relevant content in more detail. When the information is publicized or popular rumors are popularized, screenshots of relevant information on government websites are released directly, or interviews with relevant experts and government officials are made, which greatly increases the persuasiveness of the information.

The @ sign will have a positive impact on positive emotions because the People's Daily @ is generally aimed at other media accounts or some netizens who have a representative opinion on something, which not only increases influence and persuasion but also makes the public have more of a right to know and express, and feel that their views have been valued. Finally, when there is reposted content, negative emotions will be significantly reduced. The reason is that it was found in the crawled Weibo that even when reposting Weibo, it reposted its previous content, and then reported some new information and added some new progress. When a Weibo is a positive event, repeated retransmissions help to expand its influence and increase acceptance and credibility, while when Weibo is a negative event, people follow up on the progress of the event and publish the media's own views. This is conducive to guiding public opinion to develop in a good direction.

\subsubsection{Weibo Comment Characteristic Variables}

The number of followers, the number of followings, the number of Weibo posts, the level, and whether the user is authenticated represent the influence and activity of a user in Weibo all have a positive effect on emotional tendencies. The more followers a user has, the greater the user's influence. Such users will become opinion leaders within a certain range. They have their own opinions on things, are easy to accept the development of emerging things, and are more rational in looking at problems. China pays more attention to its own words and deeds, and rarely publishes excessive speech. The number of Weibos and ranks are generally proportional to the number of followers. In order to maintain their status and keep fans from losing, an opinion leader will post more updates and communicate more with other users, so the level will be higher. Among them, certified Weibo users will have a much higher number of followers, Weibos, and levels, and they have a higher probability of positive attitudes towards GM. However, compared with ordinary users, the number of authenticated users is small. They participated in fewer reviews and accounted for only $6 \%$ of all reviews. Users who pay more attention will receive more information and channels so that they will not only receive unilateral information but can take a more rational view of things.

In terms of gender, male users are more likely to have positive emotions. The study found that female users only participated in a quarter of the comments on genetically modified microblogs, and most of them were negative reviews. The probable cause is that women are more prone to thinking about problems, are easily driven by negative events and emotions, and like to let go of negative emotions. Male users prefer to participate in the discussion of genetically modified topics. They think about problems more rationally. They like to talk about problems with reason and evidence, and they will not be easily affected by others. In the end, the praise obtained by the review has a negative impact on both neutral and positive sentiment, which indicates that negative reviews are more emotional and tend to gain more recognition and drive more people with the same emotion. 


\section{Conclusions and Recommendations}

\subsection{Conclusions}

This paper presents a way to deliver sustainable development to public information. Based on the analysis, the following conclusions are provided. Frist, in terms of the types of Weibo topics, Weibo on food safety, government supervision, policy systems, and public interest types all have a negative impact on users' emotional tendencies, while popular science and rumor-type Weibo have a higher probability of positive emotional tendencies. Compared to reports on security issues, regulatory issues, etc., the number of Weibos is very few, the popularization of science is far from enough and the basic rights of the people are not guaranteed.

Second, in terms of the spread effect of Weibos, negative events and emotional tendencies will be more widely spread. Banning comments will not really solve the problem. Weibos on the positive effects of GM also lacks a real rational voice and will receive more likes.

Third, in the context of Weibo text, long content will generate more negative emotions. The use of pictures, videos and @ symbols will transmit more information about GM and increase the probability of positive emotional tendencies. Forwarding Weibos with follow-up reports on events also helps the emergence of positive emotional tendencies.

Finally, in terms of the characteristics of comments, users with high influence and high activity have a higher probability of positive emotional tendencies when commenting on them; male users are more rational and positive on GM related topics than female users.

\subsection{Recommendations}

According to the above conclusions, relevant suggestions can be made to guide and improve the public opinion of GM networks, improve the public's emotional tendency, and increase the public's acceptance of GM.

First, there are many new media like People's Daily in Weibo. In the process of public opinion dissemination, they not only report the incident but also analyze and evaluate it. Its voice will affect the public's view of the incident, and then affect the direction of public opinion. But in this process, we must follow the principle of "the first time, truthful, accurate, and equal communication" [20]. Therefore, on the topic of GM, we must increase the reporting of positive events, keep abreast of policies and scientific research developments, and strengthen information disclosure; publicize genetically modified scientific knowledge to the public in a targeted and continuous manner, clarify distorted facts in a timely manner, and speak for the rights of the people. For negative event reporting, we must be objective, accurate, and follow up relevant progress in a timely manner; when commenting, do not stir up the flames, but actively guide public opinion, pay attention to excessively extreme remarks in time, and take appropriate measures to calm public anger.

Second, the media should try to be as concise as possible when transmitting relevant reports and use accurate language to convey accurate information to the public. Use pictures, videos and other information to make the report more objective and comprehensive, attracting the public's attention and alleviating the public's emotional fluctuations. Netizens interact, listen to the voices of the people, and protect the public's rights and interests, use the retransmission function to track the incident, or continue to carry out science popularization and rumor elimination, and ultimately optimize the public opinion environment.

Third, to highlight the role of Weibo users, for authenticated users and other opinion leaders in Weibo, they are at the core of the public opinion network, have more information, are more active, and have a stronger ability to disseminate information. Although their attitude towards GM is more positive, there are few related comments. Under the "silent spiral" phenomenon, it will only lead to growing opposition. Therefore, opinion leaders must improve their literacy, actively speak out, and make rational voices stronger and stronger. In addition, science education for female users will be strengthened to improve female users' emotions. 
Lastly, a good public opinion environment is more important for the government to strengthen the supervision of genetic modification and its products and improve related policies and systems. During the research, it was found that most of the public opinion craze came from problems with food safety and government supervision, coupled with slow or lack of action by the government, resulting in aggravated negative public sentiment and lack of government credibility. Therefore, the government must start from the source, from research, experiment to market entry, and further improve the system at each level to strengthen approval and supervision. Be open and transparent in policy formulation and give the people an opportunity to express their demands; at the same time, use the media and other forces to increase information disclosure, actively carry out popular science rumors, guide the public to rational discussions, and effectively protect public rights and enhance the credibility of the government.

\subsection{Strengths and Limitations}

This paper provides a new idea for the study of genetically modified public opinion. In the context of current data mining and text analysis that have been applied to many fields, a combination of big data, emotional tendency, and model regression methods has been used to study the genetically modified public opinion in the network. Based on the relevant data obtained by the self-written Python crawler program, the public opinion situation and its influencing factors were analyzed, and the reasons for the different emotional tendencies of users on the genetically modified topics in the online social platform were explored. This study innovates the traditional research on public attitudes to genetically modified genes, expands the application field of text data mining and analysis, and provides reference and inspiration for similar research. More importantly, the research has further deepened our understanding of GM public opinion and its influencing factors, and provided suggestions for the government to guide the improvement of GM public opinion and increase the public's acceptance of GM, which is conducive to the better development of GM technology, and even the improvement of government governance level.

However, there are some limitations in this study. Because of the characteristics of online social platform data, it is not possible to conduct a comprehensive survey of public individual characteristics like traditional offline surveys (such as the age, economic status of the individual, and the degree of understanding of genetically modified knowledge), so the influencing factors are still not comprehensive. This paper explored China's Sina Weibo. As Facebook and Twitter are not accessible in China, we are currently unable to collect data on Facebook and Twitter. Future research needs to be further improved and verified.

Author Contributions: Conceptualization, Y.L. and S.Y.; methodology, Y.L.; software, X.G.; validation, J.X., M.D. and R.H.; formal analysis, J.X. and M.D.; investigation, R.H.; resources, Y.L.; data curation, X.G.; writing-original draft preparation, X.G.; writing-review and editing, Y.L. and J.X.; visualization, M.D.; supervision, S.Y.; project administration, Y.L.; funding acquisition, S.Y. All authors have read and agreed to the published version of the manuscript.

Funding: This work was funded by the Fundamental Research Funds for the Central Universities (Project No. 2662017PY046), the National Social Science Foundation of China (Project No. 13CJY104) and the Earmarked Fund for Modern Agro-industry Technology Research System (Project No. CARS-23-F01 and No. CARS-21).

Acknowledgments: We would like to acknowledge reviewers for all the valuable comments and recommendations.

Conflicts of Interest: The authors declare no conflict of interest. The funders had no role in the design of the study; in the collection, analyses, or interpretation of data; in the writing of the manuscript, or in the decision to publish the results.

\section{References}

1. Tsatsakis, A.M.; Nawaz, M.A.; Tutelyan, V.A.; Golokhvast, K.S.; Kalantzi, O.I.; Chung, D.H.; Chung, G. Impact on environment, ecosystem, diversity and health from culturing and using GMOs as feed and food. Food Chem. Toxicol. 2017, 107, 108-121. [CrossRef] [PubMed] 
2. Graham, B.; Peter, B. Farm income and production impacts of using GM crop technology 1996-2015. Gm Crop. Food 2017, 8, 156-193.

3. Huang, J.K.; Peng, B.W.; Wang, X.B. Scientists' attitudes toward agricultural GM technology development and GM food in China. China Agric. Econ. Rev. 2017, 9, 369-385. [CrossRef]

4. Huang, J.K.; Peng, B.W. Consumers' perceptions on GM food safety in urban China. J. Integr. Agric. 2015, 14, 2391-2400. [CrossRef]

5. Huang, J.K.; Wang, X.B.; Dang, H. Impacts of and attitudes toward GM technology in China: Challenges, policy and research implications. China Agric. Econ. Rev. 2017, 9, 334-339. [CrossRef]

6. Sullivan, J. China's Weibo: Is faster different? New Media Soc. 2014, 16, 24-37. [CrossRef]

7. Li, M.; Jiang, P. Study on micro-blog image of genetically modified technology. Stud. Sci. Sci. 2019, 37, 1203-1211.

8. Chen, Y.; Ma, Y.C.; Xi, B. Difficulties and disintegration factors governance of emerging technologies from the perspective of governance network Experience from China 's GM crop governance. Stud. Sci. Sci. 2018, 36, 2019-2029.

9. Bekafigo, M.A.; Stepanova, E.V.; Eiler, B.A.; Noguchi, K.; Ramsey, K.L. The Effect of Group Polarization on Opposition to Donald Trump. J. Political Psychol. 2019, 40, 1163-1178. [CrossRef]

10. Liu, Y.; Bi, J.W.; Fan, Z.P. Ranking products through online reviews: A method based on sentiment analysis technique and intuitionistic fuzzy set theory. Inf. Fusion 2017, 36, 149-161. [CrossRef]

11. Hemmatian, F.; Sohrabi, M.K. A survey on classification techniques for opinion mining and sentiment analysis. Artif. Intell. Rev. 2019, 52, 1495-1545. [CrossRef]

12. Sun, S.L.; Luo, C.; Chen, J. A Review of Natural Language Processing Techniques for Opinion Mining Systems. Inf. Fusion 2016, 36, 10-25. [CrossRef]

13. Scott, S.E.; Inbar, Y.; Wirz, C.D.; Brossard, D.; Rozin, P. An Overview of Attitudes toward Genetically Engineered Food. Annu. Rev. Nutr. 2018, 38, 459-479. [CrossRef]

14. Boccia, F.; Covino, D.; Sarnacchiaro, P. Genetically modified food versus knowledge and fear: A Noumenic approach for consumer behavior. Food Res. Int. 2018, 111, 682-688. [CrossRef] [PubMed]

15. Kaya, I.; Konar, N. Urban consumer's attitudes toward genetically modified organisms and foods in Turkey. J. Agric. Sci. (Turk.) 2014, 1, 71-82.

16. Ghasemi, S.; Karami, E.; Azadi, H. Knowledge, attitudes and behavioral intentions of agricultural professionals toward genetically modified (GM) foods: A case study in Southwest Iran. Sci. Eng. Ethics 2013, 19, 1201-1227. [CrossRef] [PubMed]

17. Han, F.; Zhou, D.; Liu, X.; Cheng, J.; Zhang, Q.; Shelton, A.M. Attitudes in China about crops and foods developed by biotechnology. PLoS ONE 2015, 10, e0139114. [CrossRef]

18. McComas, K.A.; Besley, J.C.; Steinhardt, J. Factors influencing US consumer support for genetic modification to prevent crop disease. Appetite 2014, 78, 8-14. [CrossRef]

19. Öz, B.; Unsal, F.; Movassaghi, H. Consumer attitudes toward genetically modified food in the United States: Are Millennials different? J. Transnatl. Manag. 2018, 23, 3-21. [CrossRef]

20. Bukenya, J.O.; Wright, N.R. Determinants of consumer attitudes and purchase intentions with regard to genetically modified tomatoes. Agribus. Int. J. 2007, 23, 117-130. [CrossRef]

21. Zhu, X.; Xie, X. Effects of knowledge on attitude formation and change toward genetically modified foods. Risk Anal. 2015, 35, 790-810. [CrossRef] [PubMed]

22. Lu, X.; Xie, X.; Xiong, J. Social trust and risk perception of genetically modified food in urban areas of China: The role of salient value similarity. J. Risk Res. 2015, 18, 199-214. [CrossRef]

23. Park, S.; Kim, Y. Building thesaurus lexicon using dictionary-based approach for sentiment classification. In Proceedings of the 2016 IEEE 14th International Conference on Software Engineering Research, Management and Applications (SERA), Towson, ML, USA, 8-10 June 2016; pp. 39-44.

24. Ai, Y.; Chen, Z.; Wang, S.; Pang, Y. Recognizing emotions in chinese text using dictionary and ensemble of classifiers. In Proceedings of the Third International Workshop on Pattern Recognition, Jinan, China, 26-28 May 2018; p. 1082807.

25. Liu, C.; Xu, L.; Wang, Z.; Wang, N. Sentiment analysis method based on context view. In Proceedings of the 2016 12th International Conference on Natural Computation, Fuzzy Systems and Knowledge Discovery (ICNC-FSKD), Changsha, China, 13-15 August 2016; pp. 1577-1581. 
26. Asghar, M.Z.; Khan, A.; Bibi, A.; Kundi, F.M.; Ahmad, H. Sentence-level emotion detection framework using rule-based classification. Cogn. Comput. 2017, 9, 868-894. [CrossRef]

27. De-Yang, Z.; Yi-Liang, H.; Xiao-Long, L. Research on micro-blog emotional tendency based on keyword extraction and dependency syntax. In Proceedings of the 2018 37th Chinese Control Conference (CCC), Wuhan, China, 25-27 July 2018; pp. 9654-9659.

28. Huang, L.; Li, S.; Zhou, G. Emotion corpus construction on microblog text. In Proceedings of the Workshop on Chinese Lexical Semantics, Beijing, China, 9-11 May 2015; pp. 204-212.

29. Xu, H.; Yang, W.; Wang, J. Hierarchical emotion classification and emotion component analysis on Chinese micro-blog posts. Expert Syst. Appl. 2015, 42, 8745-8752. [CrossRef]

30. Wang, L.; Hu, G.; Zhou, T. Semantic analysis of learners' emotional tendencies on online MOOC education. Sustainability 2018, 10, 1921. [CrossRef]

31. Zhu, N.; Cai, Y.-h.; Sun, F.-w.; Yang-yang, Y.-f. Mapping the emotional landscape: The role of specific emotions in conceptual categorization. Acta Psychol. 2015, 159, 41-51. [CrossRef]

32. Sun, Y.; Quan, C.; Kang, X.; Zhang, Z.; Ren, F. Customer emotion detection by emotion expression analysis on adverbs. Inf. Technol. Manag. 2015, 16, 303-311. [CrossRef]

33. Hu, N.; Koh, N.S.; Reddy, S.K. Ratings lead you to the product, reviews help you clinch it? The mediating role of online review sentiments on product sales. Decis. Support Syst. 2014, 57, 42-53. [CrossRef]

34. Topal, K.; Ozsoyoglu, G. Movie review analysis: Emotion analysis of IMDb movie reviews. In Proceedings of Proceedings of the the 2016 IEEE/ACM International Conference on Advances in Social Networks Analysis and Mining, San Francisco, CA, USA, 18-21 August 2016; pp. 1170-1176.

35. Habibi, R.; Setyohadi, D.B.; Santoso, K.I. Student learning styles and emotional tendencies detection based on Twitter. In Proceedings of the 2017 4th International Conference on Information Technology, Computer, and Electrical Engineering (ICITACEE), Jawa Tengah, Indonesia, 18-19 October 2017; pp. 239-243.

36. Nofer, M.; Hinz, O. Using Twitter to Predict the Stock Market: Where is the Mood Effect? Publ. Darmstadt Tech. Univ. Inst. Bus. Stud. 2015, 57, 63-88. [CrossRef]

37. Hernandez-Suarez, A.; Sanchez-Perez, G.; Martinez-Hernandez, V.; Perez-Meana, H.; Toscano-Medina, K.; Nakano, M.; Sanchez, V. Predicting political mood tendencies based on Twitter data. In Proceedings of the 2017 5th International Workshop on Biometrics and Forensics (IWBF), Coventry, UK, 4-5 April 2017.

38. Zhao, Y.; Qin, B.; Dong, Z.; Chen, H.; Liu, T. What causes different emotion distributions of a hot event? A deep event-emotion analysis system on microblogs. In Natural Language Processing and Chinese Computing; Springer: Piscataway, NJ, USA, 2015; pp. 453-464.

39. Kramer, A.D.; Guillory, J.E.; Hancock, J.T. Experimental evidence of massive-scale emotional contagion through social networks. Proc. Natl. Acad. Sci. USA 2014, 111, 8788-8790. [CrossRef]

40. Goel, P.; Thareja, R. Emotion Analysis of Twitter Data Using Hashtag Emotions. In Proceedings of the International Conference on Application of Computing and Communication Technologies, Nagoya, Japan, 27-30 April 2018; pp. 88-98.

41. Fan, R.; Zhao, J.; Chen, Y.; Xu, K. Anger is more influential than joy: Sentiment correlation in Weibo. PLoS ONE 2014, 9, e110184. [CrossRef] [PubMed]

(C) 2020 by the authors. Licensee MDPI, Basel, Switzerland. This article is an open access article distributed under the terms and conditions of the Creative Commons Attribution (CC BY) license (http://creativecommons.org/licenses/by/4.0/). 